\title{
A Study in User-Centered Design and Evaluation of Mental Tasks for BCI
}

\author{
Danny Plass-Oude Bos, Mannes Poel, and Anton Nijholt \\ University of Twente, Faculty of EEMCS, \\ PO Box 217, 7500 AE Enschede, The Netherlands \\ $\{$ d.plass,m.poel,a.nijholt\}@ewi.utwente.nl
}

\begin{abstract}
Current brain-computer interfacing (BCI) research focuses on detection performance, speed, and bit rates. However, this is only a small part of what is important to the user. From human-computer interaction (HCI) research, we can apply the paradigms of user-centered design and evaluation, to improve the usability and user experience. Involving the users in the design process may also help in moving beyond the limited mental tasks that are currently common in BCI systems. To illustrate the usefulness of these methods to BCI, we involved potential users in the design process of a BCI system, resulting in three new mental tasks. The experience of using these mental tasks was then evaluated within a prototype BCI system using a commercial online role-playing game. Results indicate that user preference for certain mental tasks is primarily based on the recognition of brain activity by the system, and secondly on the ease of executing the task.
\end{abstract}

Keywords: user-centered design, evaluation, brain-computer interfacing, multimodal interaction, games.

\section{Introduction}

The research field of brain-computer interfaces (BCI) originates from the wish to provide fully-paralyzed people with a new output channel to enable them to interact with the outside world, despite their handicap. As the technology is getting better, the question arises whether BCI could also be beneficial for healthy users in some way, for example, by improving quality of life or by providing private, handsfree interaction [1118. There are still a lot of issues yet to solve, such as delays, bad mental task recognition rates, long training times, and cumbersome hardware [8]. Current BCI research concentrates on improving the recognition accuracy and speed, which are two important factors of how BCI systems are experienced. On the other hand there is a lot of interest for making BCI a more usable technology for healthy users [13. But in order for this technology to be accepted by the general public, other factors of usability and user experience have to be taken into account as well [1419. There is some tentative research in this direction, such as Ko et al. who evaluated the convenience, fun and intuitiveness of a BCI game they developed [6], and Friedman 
et al. who looked into the level of presence experienced in two different navigation experiments 3]. But a lot of research still needs to be conducted.

In this paper we focus on applying HCI principles to BCI design. In Section 2 we apply a user-centered design method to the selection of mental tasks for shapeshifting in the very popular massively-multiplayer online role-playing game World of Warcraft ${ }^{\circledR}$, developed by Blizzard Entertainment, Inc ${ }^{\circledR}$. Within the large group of healthy users, gamers are an interesting target group. Fed by a hunger for novelty and challenges, gamers are often early adopters of new paradigms [12]. Besides, it is suggested that users will be able to stay motivated and focused for longer periods if the BCI experiment can be presented in a game format 4.

Afterwards, in Section 3 and 4 the selected mental tasks are evaluated in a user study; in this evaluation the focus was on the user preferences - which among others include recognition, immersion, effort, ease of use - for the designed mental tasks. The main research questions we try to answer are: Which mental tasks do the users prefer, and why? How may this preference be influenced by the detection performance of the system? The results are discussed in Section 5 and conclusions of this user-centered design and evaluation can be found in Section 6 .

\section{User-Centered Design: What Users Want}

One of the problems facing BCI research is the uncovering of usable mental tasks that trigger detectible brain activity. The tasks (by convention indicated by the name of the corresponding brain activity) that are currently most popular are: slow cortical potentials, imaginary movement, P300, and steady-state visuallyevoked potentials [1517]. Users regularly indicate that these tasks are either too slow, nonintuitive, cumbersome, or just annoying to use for control BCIs 1012. Current commercial applications are a lot more complex and offer a lot more interaction possibilities than applications used in BCI research. Whereas current game controllers have over twelve dimensions of input, BCI games are generally limited to one or two-dimensional controls. Also, the mental tasks that are available are limited in their applicability for intuitive interaction. New mental tasks are needed that can be mapped in an intuitive manner with the system action.

One way to discover mental tasks that are suitable from a user perspective is to simply ask the user what they would like to do to trigger certain actions. In World of Warcraft ${ }^{\circledR}$, the user can play an elf druid who can shape-shift into animal forms. As an elf, the player can cast spells to attack or to heal. When in bear form, the player can no longer use most spells, but is stronger and better protected against direct attacks, which is good for close combat.

In an open interview, we asked four World of Warcraft ${ }^{\circledR}$ players of varying expertise and ages what mental tasks they would prefer to use to shape-shift from the initial elf form to bear, and back again. The participants were not informed about the limits of current BCI systems, but most people did need an introduction to start thinking about tasks that would have a mental component. 
They were asked to think of using the action in the game, and what it means to them, what it means to their character in the game, what they think about when doing it, what they think when they want to use the action, and then to come up with mental tasks that fit naturally with their gameplay. The ideas that the players came up with can be grouped into three categories. For the user evaluation of these mental tasks in Section 3, these three categories were translated into concrete mental tasks, mapped to the in-game action of shapeshifting. Each category consists of a task, and its reverse, to accommodate the shapeshifting action in the directions of both bear and elf form.

1. Inner speech: recite a mental spell to change into one form or the other. The texts of spells subsequently used were derived from expressions already used in the game world. The user had to mentally recite "I call upon the great bear spirit" to change to bear. "Let the balance be restored" was the expression used to change back to elf form.

2. Association: think about or feel like the form you want to become. Concretely, this means the user had to feel like a bear to change into a bear, and to feel like an elf to change into an elf.

3. Mental state: automatically change into a bear when the situation demands it. When you are attacked, the resulting stress could function as a trigger. For the next step of this research, this had to be translated into a task that the users could also perform consciously. To change to bear form the users had to make themselves feel stressed; to shift into elf form, relaxed.

\section{User Evaluation Methodology}

The goal of the user evaluation was to answer the following questions in this game context: Which mental tasks do the users prefer, and why? How may this preference be influenced by the detection performance of the system?

Fourteen healthy participants (average age 27, ranging from 15 to 56 ; 4 female) participated in the experiment voluntarily. All but one of the participants were right-handed. Highest finished education ranged from elementary school to a master's degree. Experience with the application World of Warcraft ${ }^{\circledR}$ ranged from "I never play any games" to "I raid daily with my level 80 druid". Three participants were actively playing on a weekly basis. A written informed consent was obtained from all participants.

The general methodology to answer these questions was as follows. In order to measure the influence of the detection performance of the system the participants were divided in two groups, a so-called "real-BCI" and "utopia-BCI" group. The group that played World of Warcraft ${ }^{\circledR}$ with "utopia-BCI" decided for themselves whether they performed the mental task correctly, and pressed the button to shapeshift when they had. In this way a BCI system with $100 \%$ detection performance (an utopia) was simulated. The group that played World of Warcraft ${ }^{\circledR}$ with "real-BCI" actually controlled their shapeshifting action with their mental tasks, at least insofar as the system could detect it. 
The participants came in for experiments once a week for five weeks, in order to track potential changes over time. During an experiment, for each pair (change to bear, to elf) of mental tasks (inner speech, association, and mental state), the participant underwent a training and game session and filled in questionnaires to evaluate the user experience. The following sections explain each part of the methods in more detail.

\subsection{Weekly Sessions and Measurements}

The participants participated in five experiments, lasting about two hours each, over five weeks. The mental tasks, mentioned above, were evaluated in random order to eliminate any potential order effects, for example, due to fatigue or user learning.

For each task pair, a training session was done. The purpose of the training session was manifold: it gathered clean data to evaluate the recognizability of the brain activity related to the mental tasks, the user was trained in performing the mental tasks, the system was trained for those participants who play the game with the real BCI system, and the user experience could be evaluated outside the game context. A training session consisted of two sets of trials with a break in between during which the participant could relax. Each set started with four watch-only trials (two per mental task), followed by 24 do-task trials (twelve per mental task). The trial sequence constituted of five seconds watching the character in their start form, followed by two seconds during which the shape-shifting task was presented. After this the participant had ten seconds to perform the mental task repeatedly until the time was up, or just watch if it was a watch-only trial. At the end of these ten seconds, the participant saw the character transform. See Figure 1 for a visualization of the trial sequence. During the watch-only trials, the participant saw exactly what they would see during the do-task trials, but they were asked only to watch the sequence. This watch-only data was recorded so it could function as a baseline. It is possible that simply watching the sequence already induces certain discriminable brain activity. This data provides the possibility to test this. The character in the videos was viewed from the back, similar to the way the participant would see the avatar in the game.

At the end of the training session, the participant was asked to fill in forms to evaluate the user experience. The user experience questionnaire was loosely based on the Game Experience Questionnaire [5]. It contained statements for which the participants had to indicate their amount of agreement on a five-point Likert scale, for example: "I could perform these mental tasks easily", "It was tiring to use these mental tasks", and "It was fun to use these mental tasks". The statements can be categorized into the following groups: whether the task was easy, doable, fun, intuitive, tiring to execute, whether they felt positive or negative about the task, and whether the mapping to the in-game action made sense or not.

After the training session, the participant had roughly eight minutes to try the set of mental tasks in the game environment. The first experiment consisted only 


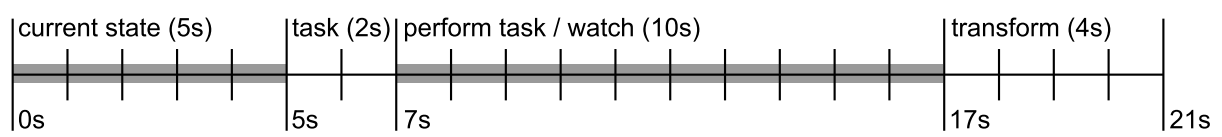

Fig. 1. Training session trial sequence: first the character is shown in their start form, then the task is presented, after which there is a period during which this task can be performed. At the end the animation for the shape-shift is shown.

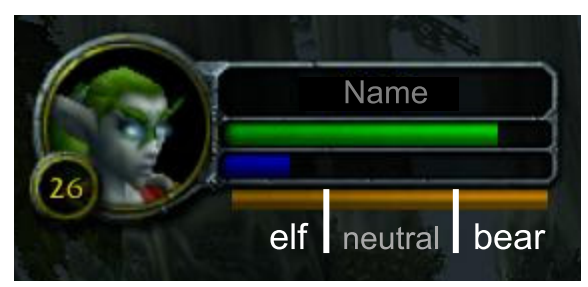

Fig. 2. Orange feedback bar with thresholds. The user has to go below 0.3 to change to elf, and above 0.7 to change to bear. In between no action is performed.

of a training session. For weeks two up to and including four, the participants were split up into the "real-BCI" and "utopia-BCI" group. Groups were fixed for the total experiment. During the last week all participants followed a training and played the game with the real BCI system.

The "real-BCI" group received feedback on the recognition of their mental tasks in the form of an orange bar in the game (see Figure 2). The smaller the bar, the more the system had detected the mental task related to elf form. The larger, the more the system had interpreted the brain activity as related to bear form. When the thresholds were crossed the shape-shift action was executed automatically.

The "utopia-BCI" group participants had to interact with a BCI system with (a near) $100 \%$ performance. Since this is not technical feasible yet, one could rely on a Wizard of Oz technique [16. The users wear the EEG cap and have to perform the mental tasks when they wanted to shapeshift and the Wizard decides when it was performed correctly. In this case, however, the Wizard would have no way of knowing what the user is doing as there is no external expression of the task. The only option left to simulate a perfect system is to let the participants evaluate themselves whether or not they had performed the task correctly. Then they pressed the shape-shift button in the game manually.

At the end of the session, the user experience questionnaire was repeated, to determine potential differences between the training and game sessions. The game session questionnaire contained an extra question to determine the perceived detection performance of the mental tasks. A final form concerning the experiment session as a whole was filled in at the end of the session. The participants were asked to put the mental tasks in order of preference, and to indicate why they choose this particular ordering. 


\subsection{EEG Analysis and Mapping}

The EEG analysis pipeline, programmed in Python, was kept very general, as there was no certainty about how to detect these selected mental tasks. Common Average Reference was used as a spatial filter, in order to improve the signalto-noise ratio [9]. The bandpass filter was set to $1-80 \mathrm{~Hz}$. The data gathered during the training session was sliced in 10-second windows. These samples were whitened [2, and the variance was computed as an indication of the power in the window. A support vector machines (SVM) classifier trained on the training session data provided different weights for each of the EEG channels.

To make the BCI control more robust to artifacts, two methods were applied to the mapping of classification results to in-game actions. A short dwelling was required to trigger the shape-shift, so it would not be activated by quick peaks in power. Secondly, hysteresis was applied: the threshold that needed to be crossed to change into a bear was higher than the threshold required to revert back to elf form. In between these two thresholds was a neutral zone in which no action was performed, see also Figure 2 .

\section{Results}

\subsection{Which Mental Tasks Do Users Prefer and Why?}

In the post-experiment questionnaire, the participants were asked to list the mental tasks in order of preference. The place in this list was used as a preference score, where a value of 1 indicated first choice, and 6 is the least preferable. These values were rescaled to match the user experience questionnaire values: ranging from 1 to 5 , with 5 most preferable, and therefore 3.0 indicates a neutral disposition in preference order. Sixty-nine measurements were obtained from 14 participants over five weeks. One week one participant had to leave early and could not fill in his preference questionnaire.

The average preference scores show a general preference for the association tasks, and the mental state seems to be disliked the most. But this paints a very simplistic image, as there are large differences between the "real-BCI" and "utopia-BCI" groups.

To better understand the effects of the different aspects, Figure 3 shows the preference and user experience scores for each of the three mental task pairs, separate for the two participant groups. Whereas for the "real-BCI" group the mental state tasks are most liked, for the "Utopia BCI" group, they are most disliked. Similarly, The "utopia-BCI" group most preferred inner speech, which was least preferred by the "real-BCI" group. Because of these large differences, these two groups need to be investigated as two separate conditions.

\subsection{What Is the Influence of Recognition Performance on Task Preference?}

Although it is not possible to completely separate the influence of the recognition performance and other aspects that differ between the participant groups, based 

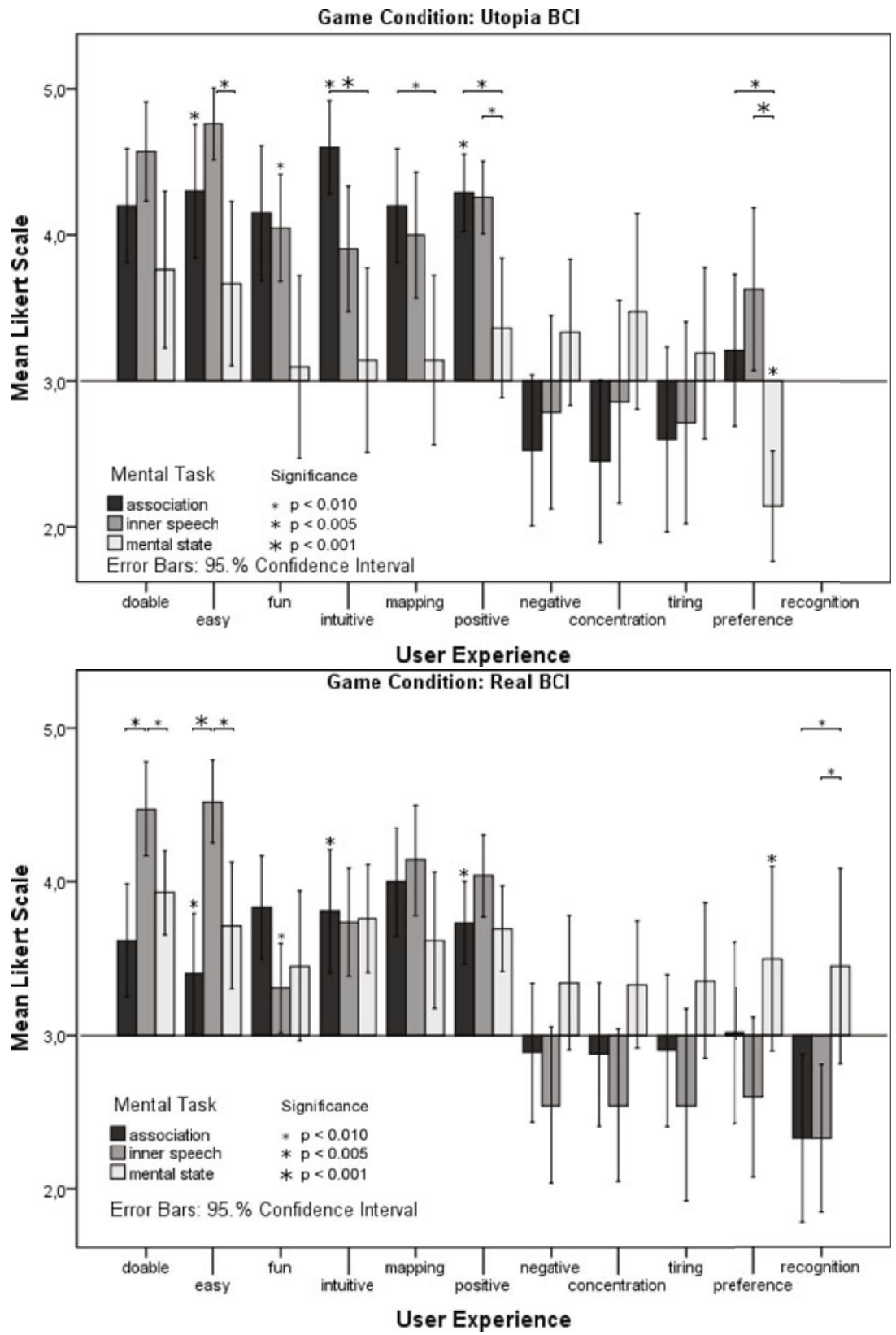

Fig. 3. User experience, preference, and perceived performance scores for the "utopiaBCI" and "real-BCI" groups, separate for the three mental task pairs, averaged for weeks 2 to 4 . The bar plot is annotated with significant differences between task pairs (association, inner speech, mental state; with a line with star above the two pairs) and game conditions ("utopia-BCI", "real-BCI"; indicated by a star above the bar). 
on the user experience scores, recognition perception scores, and the words the participants used to describe their reasoning for their preference, it is possible to explain the discrepancy between the two conditions and get an idea of the influence of recognition performance.

Inner speech is preferred by the "utopia-BCI" group, mainly because it is considered easy and doable. Although the inner speech tasks were rated highly by both groups, the system recognition had a heavy impact: it is the least preferred task pair for the "real-BCI" participants. The association tasks are valued mostly for their intuitiveness and the mapping with the in-game task, by the "utopia-BCI" group. Where the bad detection of inner speech mainly affected the preference scores, for association the user experience is significantly different on multiple aspects: easy, intuitive, and positive. The opposite happens for mental state. This task pair scores low across the board by both groups, yet it was preferred by the "real-BCI" group. It was also the task that was best recognized by the system, which is reflected in the perceived recognition scores.

Based on these results, it seems that the recognition performance has a strong influence on the user preference, which is the most important consideration for the "real-BCI" group. For the "utopia-BCI" group different considerations emerge, where the ease of the task pair seems to play a dominant role, followed by the intuitiveness.

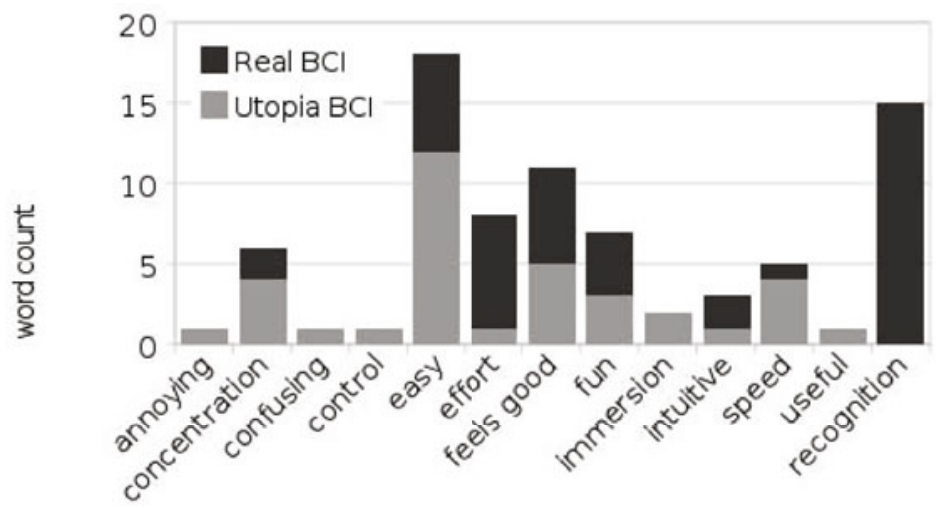

word categories

Fig. 4. Counts for the categories of words used to describe the reasoning behind the participant's preference ranking, total for weeks 2 to 4

This view is confirmed by looking at the reasons the participants describe for their preference ranking, see Figure 4. The words they used were categorized, and the number of occurrences within each category was used as an indication of how important that category was to the reasoning. To reduce the number of categories, words that indicated a direct opposite and words that indicated a similar reason were merged in one category. For example, difficult was recoded to easy, and tiring was recoded to effort. In the words used by the "real-BCI" 
group, recognition performance is the most used $(n=15)$, more than twice as any other word category $(n<=7)$. The 'Utopia BCI' group mostly referred to the ease of executing the task ( $n=12$, where $n<=5$ for the other word categories). Other issues that were often mentioned were effort, feels good, and speed.

\subsection{Correlations between Preference and User Experience}

Given the fact that participants indicated task recognition and ease to be the most important considerations for their preference, do these aspects from the user experience questionnaire also show a correlation to the preference scores?

Weeks 1 and 5 were excluded from this analysis as both groups perform the tasks in the same conditions in these weeks ("Utopia" in week 1 and "Real" in week 2). Therefore the number of samples for the correlation tests are 63 (3 weeks, 3 task pairs, 7 participants), except for one case where there are some missing samples due to a participant having to leave early. For the correlation tests with the two conditions ("Real" and "Utopia") combined, there are twice the number of samples. There are no perceived recognition scores for the "utopiaBCI" group.

Table 1. Pearson correlation coefficients and p-values for the correlation of user experience componenents and perceived recognition rate with the preference scores for the mental task pairs. Significance annotation: $p<=0,005$ in bold.

\begin{tabular}{|c|c|c|c|c|c|c|c|c|c|}
\hline Utopia $\mathrm{r}$ &,- 482 &,- 542 &,- 478 &,- 384 &,$- 395-, 579$ & 297 & ,273 & 254 & \\
\hline $\mathrm{p}$ & ,000 & ,000 & ,000 & ,002 & ,000 & 019, & ,032 & ,047, & \\
\hline Real r &,- 218 &,- 293 &,- 294 & 057 & ,079,- 189 & 201 & ,246 & 137 & $\overline{-, 316}$ \\
\hline $\mathrm{p}$ &, 095 &, 023 &, 023 &, 665 &, 147 &, 124 & ,058 &, 295 &, 014 \\
\hline All $\mathrm{r}$ &,- 360 &,- 415 &,- 391 &,- 203 &,$- 190-, 410$ &, 252 &, 259 & ,199 &,- 316 \\
\hline $\mathrm{p}$ & ,000 & ,000 & ,000 & ,025, & ,000 & ,005 & ,004 & ,028 & ,014 \\
\hline
\end{tabular}

For the "utopia-BCI" group, the expected correlations with the ease-related aspects doable and easy were found, as well as correlations with most of the other aspects. After correction for multiple tests, the correlations with concentration and tiring are not significant. There were no significant correlations of preference with any of the user experience aspects for the "real-BCI" group, but the most relevant correlation was with the perceived recognition performance by the system, which is as expected. Looking at the conditions combined, the most significant correlations are for doable, easy, fun, and positive.

The relation between preference and recognition performance is not that apparent when investigating this correlation. Yet, the correlations do show the importance of easy, expressed in how easy and how doable it is to perform the task. They also show that other aspects can be important as well, such as fun, how intuitive it is, and the mapping to the in-game action. 


\section{Discussion}

The participants were varied in both age and experience with the game. It would be interesting to investigate the influence of these aspects on the preference and user experience.

The participants in the "utopia-BCI" group had to evaluate their execution of the mental tasks themselves. This means we had no control over whether they were really performing the tasks seriously. It is unsure how well people can evaluate their own task execution and how this may affect their experience. This is a problem in any methodology that relies on self-evaluation in any part of the experiment. Nonetheless, we assume that participants really did perform the mental tasks: during the game sessions short delays were perceived where the participants seemed to pause, after which the button was pressed. For the "utopia-BCI" participants the brain activity has been recorded as well, which would have been another motivator. The recordings cannot be used to be certain about the task execution as the execution may be different for different participants, and it is unsure whether there are clear differences between the brain signals for the task pairs to begin with.

Aspects that were indicated by the participants to influence preference ratings overall, in order of number of mentions from high to low, are: ease, recognition, feels good. Certain terms could be grouped because of similarity, like ease and effort, which would make the results more clear.

As expected, the perceived recognition performance of the system was low. This fact could have a big impact on the interpretation of the scores of the "real-BCI" group. It is however interesting to note that for the mental state task, the participants did feel the system responded to their mental actions. Over time, the perceived recognition increased, and the recognition scores for the assocation tasks also rise above the neutral response. Secondly, the participants have a tendency to be optimistic about the system recognition. A score of 1 would indicate really no recognition at all, and even though the recognition for inner speech for example would have been minimal, the average rating is still above 2. This optimism about self-assessment of the level of control you have is know from other studies [7].

This analysis focused on the features derived from explicit subject reporting, to show how valuable this information can be, or at least to indicate the importance of the user in it all. This information should preferably be combined with more objective measures, such as the speed and accuracy of the BCI system mentioned earlier. The goal when evaluating BCI systems should be to obtain a complete image in order to discover where the main problems lie. If the mental task is disliked, then perhaps it might be better to look into other tasks that are suitable for the application instead of trying to gloss it over with recognition performance improvements.

It is also important to keep in mind that these findings come from a limited context: the shapeshifting task in World of Warcraft ${ }^{\circledR}$. The task itself could have had impact on what the participants value most in a mental task. It is not possible to see task preference independent of the in-game action as they are 
inherently linked. If the game is fast-paced, tasks that can be executed more quickly will be more suitable, both on a functional and experiential level. If a task matches the in-game situation it has an advantage compared to a task that is similar in all other aspects. If a task will be executed often the effort and time of execution become more important than when it is only to be done every now and then.

Finally, the point was made that healthy users will be less accepting than patients of the problems that current BCIs still struggle with. But it is important to consider that patients will also benefit from usability improvements.

\section{Conclusions}

When evaluating BCIs, current research only pays attention to task recognition performance, speed, and the derivative: bit rates. Human-computer interaction research shows that for a user to accept and value this new means of interaction other aspects may be important as well, generally summarized as usability and user experience.

For this research, we involved potential users in the design process of determining which mental tasks to use for certain actions within the application. This resulted in three categories of mental tasks that are not listed among the most frequently used tasks in current BCI applications, and which are appreciated by the users (mental state, inner speech, and association).

The three pairs of mental tasks uncovered in the design process were evaluated based on user experience with the help of our prototype system. By asking the participants about their experience, we gained new insights into what potential users like or dislike about these particular mental tasks for this BCI system and why. In the context of this experiment, user preference for mental tasks seems to be based on (in order of preference) accuracy of task recognition by the system, ease of performing the mental task, and lastly by factors such as fun, intuitiveness, and suitability for the task.

The fact that the recognition by the system was indicated to be so important to the participants in this experiment could validate the current focus of BCI research on speed and accuracy. To prove such a generalization, further research is required. However, though the speed and accuracy of detection for a given mental task can be improved with better hardware and analysis methods, the mental task itself remains the same.

When the performance of BCI systems gets better, other aspects of user experience, such as ease, fun and intuitiveness, will play a more prominent role. When designing and evaluating systems it would, both for patients and healthy users, be beneficial to take the usability and user experience into account.

\section{Acknowledgements}

We would like to thank Betsy van Dijk for her thorough feedback, and we also gratefully acknowledge the support of the BrainGain Smart Mix Programme of 
the Netherlands Ministry of Economic Affairs and the Netherlands Ministry of Education, Culture, and Science. This work was supported by the Information and Communication Technologies Coordination and Support Action "Future BNCI" within the FP7 framework, Project Number 248320.

\section{References}

1. Blankertz, B., Popescu, F., Krauledat, M., Fazli, S., Tangermann, M., Müller, K.: Challenges for Brain-Computer Interface Research for Human-Computer Interaction Applications. In: ACM CHI Workshop on Brain-Computer Interfaces for HCI and Games (2008)

2. Dornhege, G., Blankertz, B., Krauledat, M., Losch, F., Curio, G., Muller, K.: Combined optimization of spatial and temporal filters for improving brain-computer interfacing. IEEE Transactions on Biomedical Engineering 53(11), 2274-2281 (2006)

3. Friedman, D., Leeb, R., Guger, C., Steed, A., Pfurtscheller, G., Slater, M.: Navigating virtual reality by thought: what is it like? Presence: Teleoperators and Virtual Environments 16(1), 100-110 (2007)

4. Graimann, B., Allison, B., Gräser, A.: New Applications for Non-invasive BrainComputer Interfaces and the Need for Engaging Training Environments. In: BRAINPLAY 2007 Brain-Computer Interfaces and Games Workshop at ACE (Advances in Computer Entertainment), pp. 25-28 (2007)

5. IJsselsteijn, W., de Kort, Y., Poels, K., Jurgelionis, A., Bellotti, F.: Characterising and measuring user experiences in digital games. In: International Conference on Advances in Computer Entertainment Technology (2007)

6. Ko, M., Bae, K., Oh, G., Ryu, T.: A study on new gameplay based on braincomputer interface. In: Barry, A., Helen, K., Tanya, K. (eds.) Breaking New Ground: Innovation in Games, Play, Practice and Theory: Proceedings of the 2009 Digital Games Research Association Conference, Brunel University (2009)

7. Langer, E.: The illusion of control. Journal of personality and social psychology $32(2), 311-328$ (1975)

8. Lécuyer, A., Lotte, F., Reilly, R.B., Leeb, R., Hirose, M., Slater, M.: BrainComputer Interfaces, Virtual Reality, and Videogames. Computer 41(10), 66-72 (2008)

9. McFarland, D., McCane, L., David, S., Wolpaw, J.: Spatial filter selection for EEG-based communication. Electroencephalography and clinical Neurophysiology 103(3), 386-394 (1997)

10. Molina, G.: Detection of High-Frequency Steady State Visual Evoked Potentials Using Phase Rectified Reconstruction. In: 16th European Signal Processing Conference, EUSIPCO 2008 (2008)

11. Nijholt, A., van Erp, J., Heylen, D.K.J.: BrainGain: BCI for HCI and Games. In: Proceedings AISB Symposium Brain Computer Interfaces and Human Computer Interaction: A Convergence of Ideas, pp. 32-35 (2008)

12. Nijholt, A., Tan, D., Pfurtscheller, G., Brunner, C., Millán, J.d.R., Allison, B., Graimann, B., Popescu, F., Blankertz, B., Müller, K.R.: Brain-computer interfacing for intelligent systems. IEEE Intelligent Systems pp. 76-83 (2008)

13. Nijholt, A., Tan, D., Millán, J.d.R., Graimann, B., Jackson, M.: Brain-computer interfaces for HCI and games. In: Proceedings ACM CHI 2008: Art. Science. Balance, pp. 3925-3928 (2008) 
14. Plass-Oude Bos, D., Reuderink, B., van de Laar, B., Gürkök, H., Mühl, C., Poel, M., Nijholt, A., Heylen, D.: Brain-computer interfacing and games. In: Tan, D., Nijholt, A. (eds.) Brain-Computer Interfaces: Applying our Minds to Human-Computer Interaction, ch. 10. Springer, Heidelberg (2010)

15. Reuderink, B.: Games and Brain-Computer Interfaces: The State of the Art. Tech. Rep. TR-CTIT-08-81, Human Media Interaction, Faculty of EEMCS, University of Twente (2008)

16. Salber, D., Coutaz, J.: Applying the wizard of oz technique to the study of multimodal systems. Human-Computer Interaction 753, 219-230 (1993)

17. Tonet, O., Marinelli, M., Citi, L., Rossini, P., Rossini, L., Megali, G., Dario, P.: Defining brain-machine interface applications by matching interface performance with device requirements. Journal of Neuroscience Methods 167(1), 91-104 (2008)

18. Wolpaw, J.R., Loeb, G.E., Allison, B.Z., Donchin, E., Nascimento, O.F., Heetderks, W.J., Nijboer, F., Shain, W.G., Turner, J.N.: BCI meeting 2005 - workshop on signals and recording methods. IEEE Transactions on Neural System and Rehabilitation Engineering 14(2), 138-141 (2006)

19. Wolpaw, J., Birbaumer, N., McFarland, D., Pfurtscheller, G., Vaughan, T.: Brain-computer interfaces for communication and control. Clinical Neurophysiology 113(6), 767-791 (2002) 